\title{
Em hidrocefalia aguda e hematoma extradural da fossa posterior na infância é necessária a derivação?
}

\section{Relato de caso}

\author{
Carlos Umberto Pereira*, Alvino Dutra da Silva** \\ Serviço de Neurocirurgia do Hospital João Alves Filho. Aracaju, SE
}

\section{RESUMO}

O hematoma extradural traumático da fossa posterior na infância pode cursar com hidrocefalia obstrutiva aguda. Em casos de diagnóstico precoce, dependendo dos achados tomográficos e das condições neurológicas do paciente, a simples drenagem do hematoma pode solucionar o caso, não havendo necessidade de instalação de derivação ventricular peritoneal ou externa para tratar a hidrocefalia concomitante.

Os autores apresentam um caso na infância de hematoma extradural agudo da fossa posterior com extensão supratentorial, associado à hidrocefalia obstrutiva aguda. A criança foi submetida à craniectomia occipital unilateral, com resultado excelente. A tomografia pós-operatória demonstrou a regressão da hidrocefalia. Não houve necessidade de derivação ventricular.

\section{PALAVRAS-CHAVE}

Hematoma extradural de fossa posterior. Hidrocefalia.

\section{ABSTRACT}

Acute hydrocephalus with posterior fossa extradural hematoma in infancy. Is the shunting necessary? Case report

Pediatric traumatic epidural hematoma of posterior fossa may cause acute ventricular obstruction Early diagnosis and evacuation of the hematoma may preclude the need of external or internal ventricular shunting.

We report a case of epidural hematoma of posterior fossa with supratentorial extension and hydrocephalus in a 5 year-old child. The treatment consisted in a unilateral occipital craniectomy with excellent outcome. The postoperative CT confirmed the resolution of the hydrocephalus without ventricular shunting.

\section{KEYWORDS}

Posterior fossa epidural hematoma. Hydrocephalus.

\section{Introdução}

Hematomas traumáticos da fossa posterior são considerados $\operatorname{raros}^{4,6,9,10,12,13}$. A presença de hidrocefalia aguda ocasionada por lesão expansiva traumática da fossa posterior tem sido pouco relatada na literatura médica nacional. $\mathrm{O}$ tratamento geralmente consiste na drenagem do hematoma e na derivação ventricular externa ou ventriculoperitoneal em casos de hidrocefalia obstrutiva associada ${ }^{1,8,11}$.

Os autores apresentam um caso de hidrocefalia aguda em uma criança com 5 anos de idade, portadora de hematoma extradural da fossa posterior com extensão supratentorial, que foi submetida à craniectomia suboccipital unilateral e drenagem do hematoma com resolução da dilatação ventricular, não havendo

*Professor Adjunto Doutor do Departamento de Medicina da UFS.

**Neurocirurgião do Serviço de Neurocirurgia do Hospital João Alves Filho de Aracaju. 
necessidade de derivação ventricular externa ou peritoneal, com resultado excelente.

\section{Relato do caso}

M.N.S.S., sexo feminino, 5 anos de idade. História de queda de cima de uma árvore. Deu entrada no Serviço de Emergência do Hospital João Alves Filho (Aracaju, SE) sonolenta, com vômitos, eupnéica, pupilas isocóricas, sem déficit neurológico focal. A radiografia simples do crânio mostou fratura linear occipital direita. A tomografia computadorizada mostrou lesão hiperdensa na fossa posterior direita com extensão supratentorial e hidrocefalia supratentorial (Figura 1).
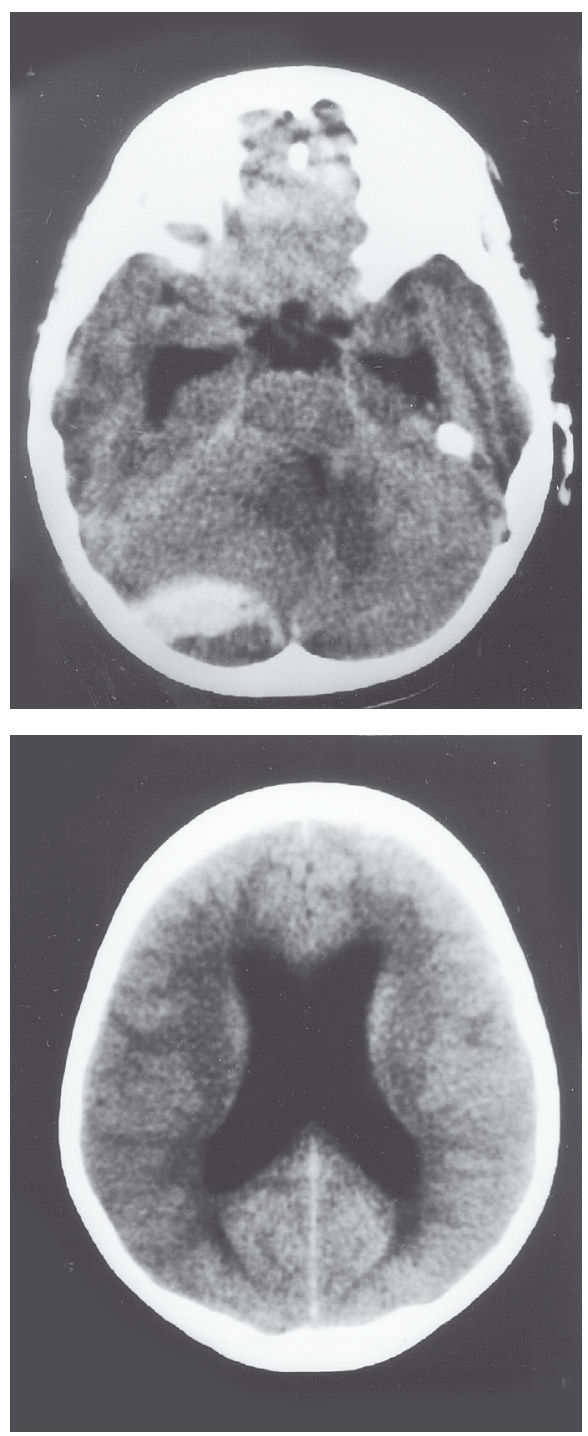

Figura 1 - TC pré-operatória apresentando lesão hiperdensa na fossa posterior direita dilatação ventricular supratentorial.
Foi submetida à craniectomia occipital direita e drenagem do hematoma. Vinte e quatro horas após o procedimento cirúrgico estava desperta, sendo submetida à nova tomografia que demonstrou ausência do hematoma na fossa posterior e supratentorial e tamanhos normais dos ventrículos (Figura 2). Recebeu alta hospitalar após 5 dias, sem déficit neurológico.

\section{Discussão}

A incidência de hematoma extradural da fossa posterior na infância varia de $2 \%$ a $4 \%$ de todas as complicações por traumatismo craniencefálico ${ }^{3,5}$. Vários autores relatam que a incidência de hidrocefalia aguda
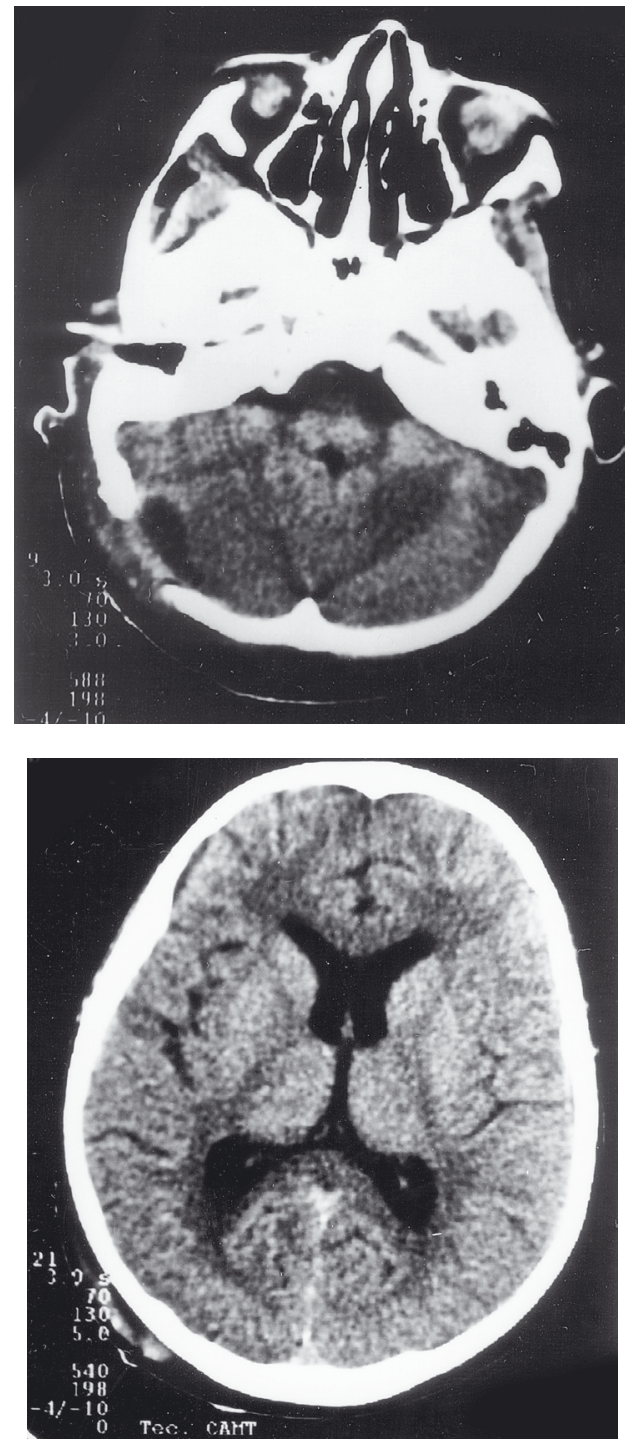

Figura 2 - TC pós-operatória demonstrando ausência de hematoma na fossa posterior e ventrículos supratentoriais de tamanho e simetria normais. 
em casos de hematoma extradural da fossa posterior varia de $13,3 \%$ a $61,1 \%{ }^{2,7}$.

O hematoma extradural traumático da fossa posterior na infância tem uma tendência maior a ficar restrito apenas na fossa posterior, enquanto no adulto pode estender-se para a região supratentorial ${ }^{6}$. A maioria dos casos é de localização unilateral ${ }^{6,8}$. Segundo Karasawa e cols. ${ }^{3}$, em sua série existe uma relação significativa entre os casos de hematoma extradural da fossa posterior com extensão supratentorial e hidrocefalia, quando o hematoma apresenta espessura de $15 \mathrm{~mm}$ ou mais, bem como anormalidades das cisternas mesencefálicas. Segundo esses autores, hematoma extradural da fossa posterior que se estende para a região supratentorial comprime o aqueduto de Silvius posteriormente e ocasiona hidrocefalia obstrutiva, fato este observado em nosso paciente

Neubauer $^{8}$ recomenda, nesses casos, drenagem cirúrgica do hematoma associada à derivação ventricular externa. Canoni e cols. ${ }^{1}$ relatam sua experiência em 21 casos de hematomas traumáticos da fossa posterior em que quatro pacientes apresentaram hidrocefalia e necessitaram de derivação ventricular externa. Karasawa e cols. ${ }^{3}$ demonstraram tomograficamente que o quadro de hidrocefalia apresenta resolução imediata após drenagem do hematoma, sem necessidade de instalação de drenagem ventricular externa, com o que estamos de acordo, conforme resultado em nosso paciente.

Como ficou demonstrado neste caso, em hematoma traumático da fossa posterior associado à hidrocefalia obstrutiva aguda pode ocorrer resolução da hidrocefalia apenas com drenagem do hematoma, não havendo necessidade de instalação de derivação ventricular externa ou peritoneal.

\section{Referências}

1. CANNONI LF, VEIGA JCE, PERES CMA, CARVALHO RRD: Hematomas traumáticos da fossa posterior. (ferimentos não penetrantes ). J Bras Neurocirurg 8: 67-72, 1997.
2. HOLZSCHUH M, SCHUKNECHT B. Traumatic epidural haematomas of the posterior fossa: 20 new cases and a review of the literature since $1961 . \mathrm{Br} J$ Neurosurg 3:171-80, 1989.

3. KARASAWA H, FURUYA H, NAITO H, SUGIYAMA K, UENO J, KIN H: Acute hydrocephalus in posterior fossa injury. J Neurosurg 86:629-32, 1997.

4. LUI T, LEE S, CHANG C, CHENG W: Epidural hematomas in the posterior cranial fossa. J Trauma 34:211-5, 1993.

5. MOHANTY A, KOLLURI VRS, SUBBAKRISHMA DK, SATISH S, MOULI BAC, DAS BSL: Prognosis of estradural hematomas in children. Pediatr Neurosurg 23:57-63, 1995.

6. MORI K, HANDA J, MUNEMITSU H, HASHIMOTO N KOJIMA N: Epidural hematomas of the posterior fossa in children. Child' s Brain 10:130-40, 1983.

7. NAGAMINE Y, SONOBE M, TAKAHASHI S: Investigation of epidural hematomas of the posterior fossa according do CT findings. No Shinkei Geka 9:35763, 1979.

8. NEUBAUER UJ: Extradural haematoma of the posterior fossa. Twelve years experiences with CTscan. Acta Neurochir (Wien) 87:105-11, 1987.

9. PEREIRA CU, SILVA AD, LEÃO JDBC, SANTOS VBO, SANTOS MBO: Extradural hematomas of posterior fossa in childhood. Consideration about tem cases. Rev Chil Neurocirug 17:34-5, 2001.

10. PEREIRA CU, SILVA AD, LEÃO JDBC: Hematoma extradural da fossa posterior na infância. In Pereira CU, Aguiar PHP, Ramina R (eds): Tópicos em Neurocirurgia. Rio de Janeiro, Revinter, 2001, pp 167-9.

11. SOKOL JH, ROWED DW: Traumatic intracerebellar haematoma. Surg Neurol 10:340-1, 1978.

12. WRIGHT RL: Traumatic hematomas of the posterior cranial fossa. J Neurosurg 25:402-9, 1966.

13. ZUCCARELLO M, ANDRIOLI GC, FIORE DL. LONGATTI PL, PARDATSCHER K, ZAMPIERI P: Traumatic posterior fossa haemorrhage in children . Acta Neurochir (Wien) 62:79-85, 1982.

Original recebido em fevereiro de 2002

Aceito para publicação em junho de 2002

\section{Endereço para correspondência:}

Prof. Dr. Carlos Umberto Pereira

Av. Augusto Maynard, 245/404

CEP 49015-380 - Aracaju, SE

E-mail:umberto@infonet.com.br 